\title{
HIGH SENSITIVITY C - REACTIVE PROTEIN AS A PROGNOSTIC MARKER IN
} ACUTE STROKE

Jayachandra ${ }^{1}$, Mamatha T. R², Veeranna Gowda K. M, Sowrabha Shankar Bhat ${ }^{4}$, Raghavendra ${ }^{5}$, Yashavanth Gangadkar6

\section{HOW TO CITE THIS ARTICLE:}

Jayachandra, Mamatha T. R, Veeranna Gowda K. M, Sowrabha Shankar Bhat, Raghavendra, Yashavanth Gangadkar. "High Sensitivity C-Reactive Protein as a Prognostic Marker in Acute Stroke". Journal of Evolution of Medical and Dental Sciences 2014; Vol. 3, Issue 10, March 10; Page: 2675-2679,

DOI: $10.14260 /$ jemds/2014/2191

ABSTRACT: OBJECTIVES: To determine raised serum hsCRP levels, an established risk marker in stroke patients and also to study the co relation between raised serum uric acid and hsCRP levels in ischemic and hemorrhagic stroke. MATERIALS AND METHODS: This was a cross sectional study of 200 patients presenting with a history of focal neurological deficit of acute onset in the form of hemiparesis, hemiplegia having evidence of presence of ischemic or hemorrhagic infarct in CT scan of brain. In all patients hsCRP levels were measured within 48hours of presentation. RESULTS: Most of the patients $(65 \%)$ were in the age group of more than 50 years. Left-sided hemiparesis with altered sensorium and UMN facial palsy was the most common presenting symptom. hsCRP levels were found to be increased in hemorrhagic stroke patients than with ischemic stroke, this difference was found to be statistically significant $(\mathrm{p}<0.001)$. CONCLUSION: From this study we concluded that hsCRP level is increased in cases of stroke - ischemic as well as hemorrhagic, but more in hemorrhagic stroke suggesting an inflammatory response in acute stroke. Furthermore, the increased levels correlated with larger bleed and infarct size, severe neurological deficit and worse outcome.

KEYWORDS: Ischemic stroke, hemorrhagic stroke, hsCRP level.

INTRODUCTION: Stroke is considered as a potential life threatening event in neurological patients. It is one of the leading causes of morbidity and mortality worldwide. ${ }^{1}$ This neurological deficit evolves over a few hours, persists for more than 24 hours, and is presumed to be due to an impairment of the blood supply to a part of brain.

C-reactive protein (CRP) is a glycoprotein produced by the liver, it is an acute phase reactant which is normally absent in the blood. ${ }^{2}$ The presence of acute inflammation with tissue destruction within the body stimulates its production. The CRP typically rises within 6 hours of the start of inflammation, allowing the inflammation to be confirmed. There are two types of CRP, which could be measured. The standard CRP is used to assess activity of inflammation in such chronic conditions such as arthritis; to assess for a new infection; and to monitor response to treatment of these conditions. The other type of CRP is high-sensitivity CRP (hs-CRP). This substance is considered a marker of low-grade vascular inflammation, which is a key factor in the development and rupture of atheromatous plaque.3,4

Elevated plasma levels of CRP are not disease specific but are sensitive markers which are produced in response to tissue injury, infectious agents, and inflammation. hsCRP predicts the first cardiovascular event in several populations. It was the only inflammatory marker that independently predicted the risk ${ }^{2,3}$ of stroke. hsCRP level when measured prior to the onset of clinical diseases may be an independent predictor of the first ischemic stroke. ${ }^{4}$ Our study was planned to study the high sensitivity C-reactive protein (hsCRP) level in Indian patients with acute cerebrovascular accidents. 
MATERIAL AND METHODS: This was a cross sectional study conducted for a period of 12 months from November 2012 to November 2013. 200 patients of acute stroke admitted to the emergency ward, Department of Medicine, Victoria Hospital, Bangalore were recruited. Patients presenting with history of focal neurological deficit of acute onset in the form of hemiparesis, hemiplegia or aphasia within 48hours of onset, with evidence of the presence of ischaemic or haemorrhagic stroke on CT scan of the brain were included in this study. Patients diagnosed with gout, and patients taking drugs that are known to raise serum uric acid levels like diuretics, chemotherapeutic agents, nicotinic acid, ACE inhibitors like losartan and Patients with other recognized risk factors known to increase serum uric acid levels like renal failure, leukemia, lymphoma, haemolytic anaemia, psoriasis, hypoparathyroidism were excluded.The presence of baseline vascular risk factors, including hypertension, diabetes mellitus, hypercholesterolemia, obesity, and smoking, was determined. The blood samples were then collected within 48hours of presentation and routine hematology, biochemistry tests were done. hs-CRP levels and serum uric acid levels were determined. A hS-CRP level of $=$ or $>3 \mathrm{mg} / \mathrm{L}$ was used as cut off value to determine normal or increased levels.

RESULTS: Out of 200 patients (135males and 65females) of stroke, 192 patients had CT evidence of either ischemia (116 patients) or hemorrhage (76 patients). In the remaining 8 cases, 4 cases showed diffuse atrophy \& 4 cases were normal CT study. $67.5 \%$ patients were males and $32.5 \%$ were females; (Table. 1). Maximum number of patients was in age group 41 - 70 years (mean age was 58years) (Table. 2). The most prevalent risk factor was hypertension, followed by diabetes and dyslipidemia (Table.3). 55\% of them were hypertensive and $41 \%$ were diabetic. 64 patients presented with hemiplegia, while 136 had hemiparesis. (Table.4) The most common presenting symptom was altered sensorium with left-sided weakness and UMN facial palsy. 91\% had an hsCRP level of $>$ or $=$ $3.0 \mathrm{mg} / \mathrm{L}$ and $9 \%$ had hsCRP levels $<3 \mathrm{mg} / \mathrm{L}$ in hemorrhagic stroke. hS CRP level in hemorrhagic and ischemic stroke was $7.66 \pm 2.58$, and $5.8 \pm 2.42$ respectively, which was statistically significant (Table.6). This relationship was statistically significant value $(\mathrm{P}<0.001)$.

\begin{tabular}{|c|c|c|}
\hline Gender & Number of patients & $\mathbf{\%}$ \\
\hline Male & 135 & 67.5 \\
\hline Female & 65 & 32.5 \\
\hline Total & $\mathbf{2 0 0}$ & $\mathbf{1 0 0 . 0}$ \\
\hline
\end{tabular}

Table 1: Gender distribution of patients studied

\begin{tabular}{|c|c|c|}
\hline Age in years & Number of patients & $\mathbf{\%}$ \\
\hline $17-20$ & 4 & 2.0 \\
\hline $21-30$ & 12 & 6.0 \\
\hline $31-40$ & 20 & 10.0 \\
\hline $41-50$ & 34 & 17.0 \\
\hline $51-60$ & 36 & 18.0 \\
\hline $61-70$ & 41 & 20.5 \\
\hline $71-80$ & 34 & 17.0 \\
\hline $81-90$ & 18 & 9.0 \\
\hline
\end{tabular}


ORIGINAL ARTICLE

\begin{tabular}{|c|c|c|}
\hline 91-100 & 1 & 0.5 \\
\hline Total & $\mathbf{2 0 0}$ & $\mathbf{1 0 0 . 0}$ \\
\hline \multicolumn{2}{|c|}{ Table 2: Age distribution of patients studied } \\
\hline
\end{tabular}

Mean \pm SD: $58.01 \pm 17.22$

\begin{tabular}{|l|c|c|}
\hline Comorbid conditions & $\begin{array}{c}\text { Number of patients } \\
(\mathbf{n = 2 0 0 )}\end{array}$ & $\mathbf{\%}$ \\
\hline Hypertension & 110 & 55.0 \\
\hline DM & 82 & 41.0 \\
\hline
\end{tabular}

Table 3: Comorbid conditions

\begin{tabular}{|l|c|c|}
\hline Clinical diagnosis & $\begin{array}{c}\text { Number of patients } \\
(\mathbf{n = 2 0 0 )}\end{array}$ & $\mathbf{\%}$ \\
\hline Hemiplegia & 64 & 32.0 \\
\hline Hemiparesis & 136 & 68.0 \\
\hline
\end{tabular}

Table 4: Clinical diagnosis

\begin{tabular}{|l|c|c|}
\hline CT brain findings & $\begin{array}{c}\text { Number of patients } \\
\text { (n=200) }\end{array}$ & $\mathbf{\%}$ \\
\hline Hemorrhage & 76 & 38.0 \\
\hline Infarcts & 116 & 58.0 \\
\hline Diffuse atrophy & 4 & 2.0 \\
\hline Normal Study & 4 & 2.0 \\
\hline
\end{tabular}

Table 5: CT brain findings

\begin{tabular}{|c|c|c|c|c|c|}
\hline \multirow{2}{*}{ Hs CRP } & \multicolumn{3}{|c|}{ CT brain findings } & \multirow{2}{*}{ Total } \\
\cline { 2 - 5 } & Hemorrhage & Infarcts & Diffuse atrophy & Normal Study & \\
\hline$<3.0 \mathrm{mg} / \mathrm{L}$ & $7(9.2 \%)$ & $47(40.5 \%)$ & $1(25 \%)$ & $2(50 \%)$ & $57(28.5 \%)$ \\
\hline = or $>3.0 \mathrm{mg} / \mathrm{L}$ & $69(90.8 \%)$ & $69(59.5 \%)$ & $3(75 \%)$ & $2(50 \%)$ & $143(71.5 \%)$ \\
\hline Total & $\mathbf{7 6 ( 1 0 0 \% )}$ & $\mathbf{1 1 6 ( 1 0 0 \% )}$ & $\mathbf{4 ( 1 0 0 \% )}$ & $\mathbf{4 ( 1 0 0 \% )}$ & $\mathbf{2 0 0 ( 1 0 0 \% )}$ \\
\hline \multicolumn{6}{|c|}{ Table 6: Levels of hs CRP in patients studied } \\
\hline
\end{tabular}

DISCUSSION: Stroke is the third leading cause of mortality in the western world and also a major cause of disability. Recently, it was shown that elevated CRP levels independently predict the risk of future stroke and transient ischemic attack in the elderly. 5 To analyze the role of hsCRP in stroke as a prognostic marker, the present study was undertaken. It was conducted on 200 stroke patients, among them, 116 were cases of ischemic stroke and the other 76 were cases of hemorrhagic stroke. The age distribution of the patients in this study was between 17 and 100 years. Mean age was 58 years. The risk of stroke increased with increasing age as was found in the present study. These findings were in corroboration with a study by Bamfordet $\mathrm{al}^{6}$. Besides old age, Hypertension (55\%) was the most common risk factor found in various study populations, followed by diabetes mellitus (32.5\%). Kannel ${ }^{7}$ reported that diabetes doubles the risk of stroke. Benson and Sacco ${ }^{8}$ observed that hypertension confers a relative risk for stroke of 3- to 5-fold. 
The most common presentation in the present study was altered sensorium with left-sided hemiparesis and UMN facial palsy (45\%).When the hsCRP levels were measured within 48hours of admission, it was found to be high in cases of stroke. Similar observations have been reported by various other workers also. Di Napoli ${ }^{9}$ et al in their study included 128 patients. The CRP values within 24 hours and between 48 to 72 hours were 1.3 (0.5 to 3.3) and 1.0 (0.5 to 2.3) $\mathrm{mg} / \mathrm{dl}$ respectively. Arenillas 10 et al, in their study showed that a high-sensitivity CRP level above the receiver operating characteristic curve cut-off value of $1.41 \mathrm{mg} / \mathrm{dl}$ emerged as an independent predictor of new end-point events $(\mathrm{p}<0.0001)$.

When hs CRP levels were compared in different types of stroke, the mean hsCRP level was more in hemorrhagic than ischemic stroke. These results are different from those of Yoshiyuki Wakugawa ${ }^{11}$ et al in the Hisayama study in which they observed no clear association between hsCRP levels and hemorrhagic stroke occurrence. This may be due to the presence of some confounding factors like obesity, elderly age, or due to large size of the bleed in our study secondarily leading to ischemia, and thus increasing the hsCRP level. On comparing hsCRP levels with other risk factors, no significant correlation was found. Earlier reports in the available literature have not commented regarding correlation of hsCRP level with other risk factors.

In both ischemic and hemorrhagic stroke, higher CRP concentration correlates with severe neurological deficit. This finding was similar to observations by Guo ${ }^{12}$ et al in which they had observed higher concentrations on admission correlated with leucocyte count and blood glucose level, larger infarct, severe neurological deficit and worse outcome. Kerstin Winbeck ${ }^{13}$ et al observed that an increase in CRP level between 12 and 24 hours after the onset of symptoms predicts an unfavorable outcome and is associated with an increase in the incidence of cerebrovascular and cardiovascular events. It was also seen that patients who expired had high hsCRP levels than those who survived both types of stroke. Thus, there was a relation between the hsCRP level and mortality. Higher the hsCRP level more is the chance of mortality.

CONCLUSION: From this study we conclude that hsCRP levels are increased in cases of stroke ischemic as well as hemorrhagic, but more in hemorrhagic stroke suggesting more severe inflammatory response. Furthermore, the increased levels correlated with larger volume bleed and infarct size, severe neurological deficit, and worse outcome. As we had a small sample size, a larger study is needed to endorse our observations, and to analyze further about association between hsCRP level and hemorrhagic stroke occurrence. A larger study should also focus on whether hsCRP level needs to be included as a health screening protocol.

\section{REFERENCES:}

1. Pearson TA, Mensah GA, Alexander RW. Marker of inflammation and cardiovascular diseases: application to clinical and public health practice. A statement for healthcare profession from the Centres for Disease Control and Prevention and the American Heart Association. Circulation 2003; 107: 499-511.

2. Ridker PM, Rifai N, Rose L. Comparison of C-reactive protein and low-density lipoprotein cholesterol levels in the prediction of first cardiovascular events. N Engl J Med 2002; 347 (20): 1557-65.

3. Ridker PM, Buring JE, Shih J. Prospective study of C-reactive protein and the risk of future cardiovascular events among apparently healthy women. Circulation 1998; 98 (8): 731-3. 
4. Ridker PM, Cushman M, Stampfer MJ. Inflammation, aspirin and the risk of cardiovascular disease in apparently healthy men. N Engl J Med 1997; 336 (14): 973-9.

5. Rost NS, Wolf PA, Kase CS et al. Plasma concentration of $\mathrm{C}$ reactive protein and risk of ischaemic stroke and transient ischemic attack: the Framingham study. Stroke 2001; 32(11): 2575-9.

6. Bamford J, Sandercock PAG, Dennis M et al. Classification of natural history of clinical identifiable subtypes of cerebral infarction. Lancet 1991; 337: 1521.

7. Kannel, McGreen. Diabetes most common risk factor. Arch Inern Med 1979; 54: 312-424.

8. Benson RT, Sacco RL. Stroke prevention: hypertension, diabetes, tobacco, and lipids. Neurol Clin 2000; 18 (2): 309-19.

9. Di Napoli M, Papa F, Bocola V. C-reactive protein in ischaemic stroke: an independent prognostic factor. Stroke 2001; 32:917-24.

10. Arenillas JF, Alvarez-Sabin J, Molina CA. C-reactive protein predicts further ischaemic events in first transient ischemic attack or stroke patients with intracranial large artery occlusive disease. Stroke 2003; 34 (10): 2463-8.

11. Yoshiyuki Wakugawa, Yutaka Kiyohara, Yumihiro Tanizaki et al. C-reactive protein and risk of first ever ischemic and haemorrhagic stroke in a general Japanese population. Stroke 2006; 37: 27-32.

12. Guo $\mathrm{Y}$, Jiang $\mathrm{X}$, Chen $\mathrm{S}$ et al. C-reactive protein as an important prognostic marker for ischemic stroke. Zhonghua Yu Fang Yi XueZaZhi 2003; 37: 102-4.

13. Kerstin Winbeck, Hogler Poppert, Thorleif Etgen et al. Prognostic relevance of early serial Creactive protein measurements after first ischemic stroke. Stroke 2002; 33:2459-64.

\section{AUTHORS:}

1. Jayachandra

2. Mamatha T. R.

3. Veeranna Gowda K. M.

4. Sowrabha Shankar Bhat

5. Raghavendra

6. Yashavanth Gangadkar

\section{PARTICULARS OF CONTRIBUTORS:}

1. Associate Professor, Department of Medicine, Bangalore Medical College and Research Institute, Bangalore.

2. Post Graduate Department of Medicine, Bangalore Medical College and Research Institute, Bangalore.

3. Professor, Department of Medicine, Bangalore Medical College and Research Institute, Bangalore.

4. Post Graduate, Department of Medicine, Bangalore Medical College and Research Institute, Bangalore.
5. Assistant Professor, Department of Medicine, Bangalore Medical College and Research Institute, Bangalore.

6. Post Graduate, Department of Medicine, Bangalore Medical College and Research Institute, Bangalore.

\section{NAME ADDRESS EMAIL ID OF THE CORRESPONDING AUTHOR:}

Dr. Mamatha T. R,

Postgraduate, Department of Medicine,

Bangalore Medical College and Research Institute,

Bangalore - 2

E-mail: dr.mamatha87@gmail.com

Date of Submission: 24/01/2014.

Date of Peer Review: 25/01/2014.

Date of Acceptance: 10/02/2014.

Date of Publishing: 10/03/2014. 